\title{
INJECTION TECHNIQUE FOR STUDYING RETINAL BLOOD VESSELS*†
}

\author{
BY
}

H. R. HAUSLER AND T. M. SIBAY

From the Departments of Physiology and Ophthalmology, University of Toronto, Canada

A vascular injection technique with silver nitrate (Hausler and Sibay, 1959) allows the retinal vascular tree to be seen in its full continuity and also shows up structural details of the vessel walls. By using a mixture of two silver salt solutions, this technique has now been considerably simplified, giving equally good results.

\section{Procedure}

The enucleated human eye is injected through the central retinal vessels. The lumina of these vessels are quite small, having a diameter of approximately $0.2 \mathrm{~mm}$. Two instruments which considerably facilitated their cannulation have therefore been designed. The first (Fig. 1) consists of a metal cup made by hollowing a half segment of a metal ball. This is fitted on to a metal cylinder so that it forms a universal joint, which may be moved and locked in any position.

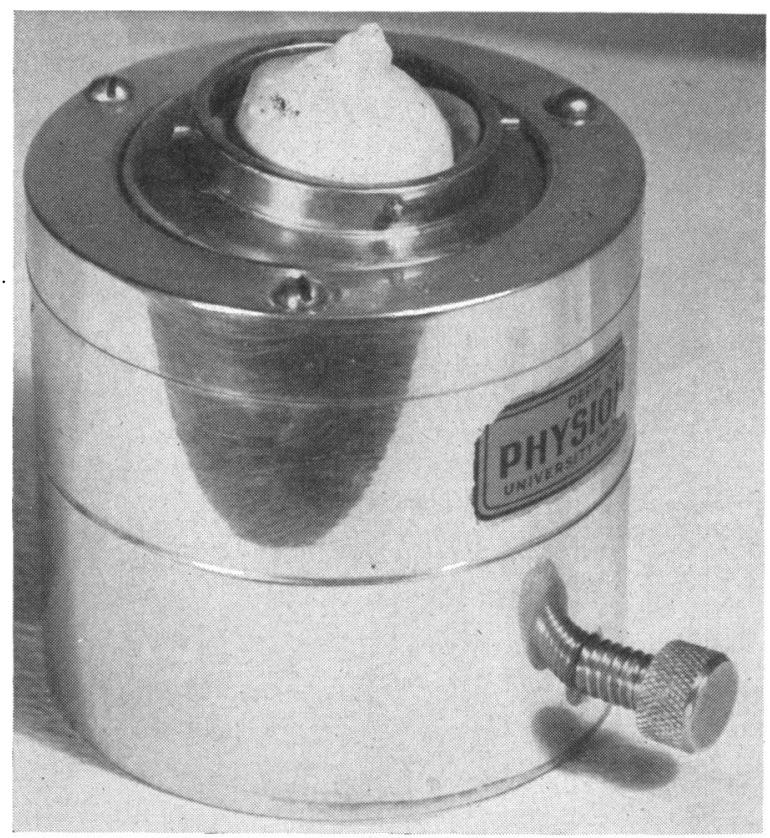

FIG. 1.-For injection of the central retinal vessels of the enucleated human eye, this instrument is used to bring the specimen into the desired position. The metal cup fitted into the base forms a universal joint, which can be locked by a turn of the screw visible near the bottom of the cylinder.

* Received for publication May 25, 1959.

† These studies were carried out under National Health Graht No. 605-9-201. 
The second piece of apparatus (Fig. 2) is a micromanipulator which allows a

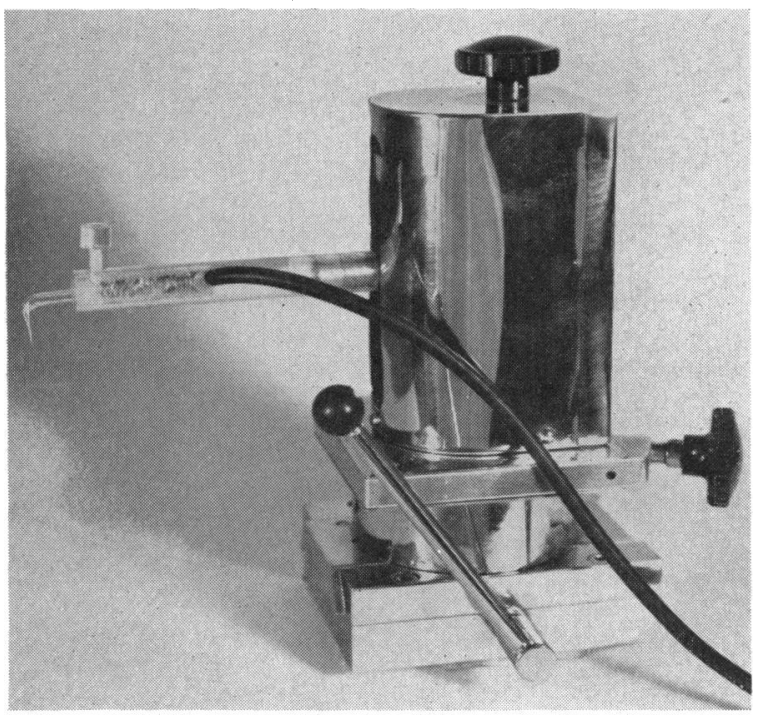

hollow plastic arm to move in any direction desired. A rubber tube is inserted into this arm and connected near the end to a fine curved glass cannula, which may be locked to the instrument by a plastic screw.

FIG. 2.-Micromanipulator used for inserting the cannula into the central retinal vessels. The plastic arm holding the glass cannula can be freely moved in three dimensions by manipulating the adjustment screws.

When it is desired to cannulate the central retinal vessels, the eye is tied into the metal cup, and this is then turned to the desired position. A drop of 10 per cent. formol saline is placed on the freshly-cut surface of the optic nerve. The central retinal vessels are observed with a dissecting microscope, and the glass cannula may be inserted into either the artery or the vein by adjusting the screws on the micromanipulator.

In animal experiments the site of cannulation will largely depend on the size of the species used. In small rodents the cannula is tied into the descending aorta, the tip pointing toward the heart. In the larger laboratory species either the internal carotid or the ophthalmic artery is cannulated.

After successful insertion of the cannula, the retinal vessels are flushed free from blood with saline, which is in turn washed out by a 10 per cent. formalin solution. This is immediately followed by injection of a 2 in 1 mixture of a 2 per cent. solution of silver dinaphthylmethane disulphonate* and a 1 per cent. solution of silver nitrate. The injected retina is then kept moist and exposed to ultra-violet light (black ray, long wave ultra-violet) for 3 to 5 minutes. The exposure to ultraviolet light is essential in this technique in order to bring out as fully as possible the structural details of the vessel walls. The intensity of the stain is influenced by the period of exposure. The eye may finally be washed free from superfluous silver solution by injecting distilled water.

The retinae are viewed as flat preparations, suspended in 10 per cent. Formalin between glass slides. Permanent preparations may be obtained by subjecting the tissue to the usual clearing and mounting procedure.

The technique stains neurofibrils, intercellular cement substances, and the perimysium of smooth muscle cells surrounding the arteriolar walls.

Typical examples are shown in Figs 3 to 7 (overleaf).

* Commercially available as "Viacutan" produced by Ward Blenkisop \& Co., 6 Henrietta Place, London, W.1. 
Fig. 3.-The region of the ora serrata in an injected human retina.
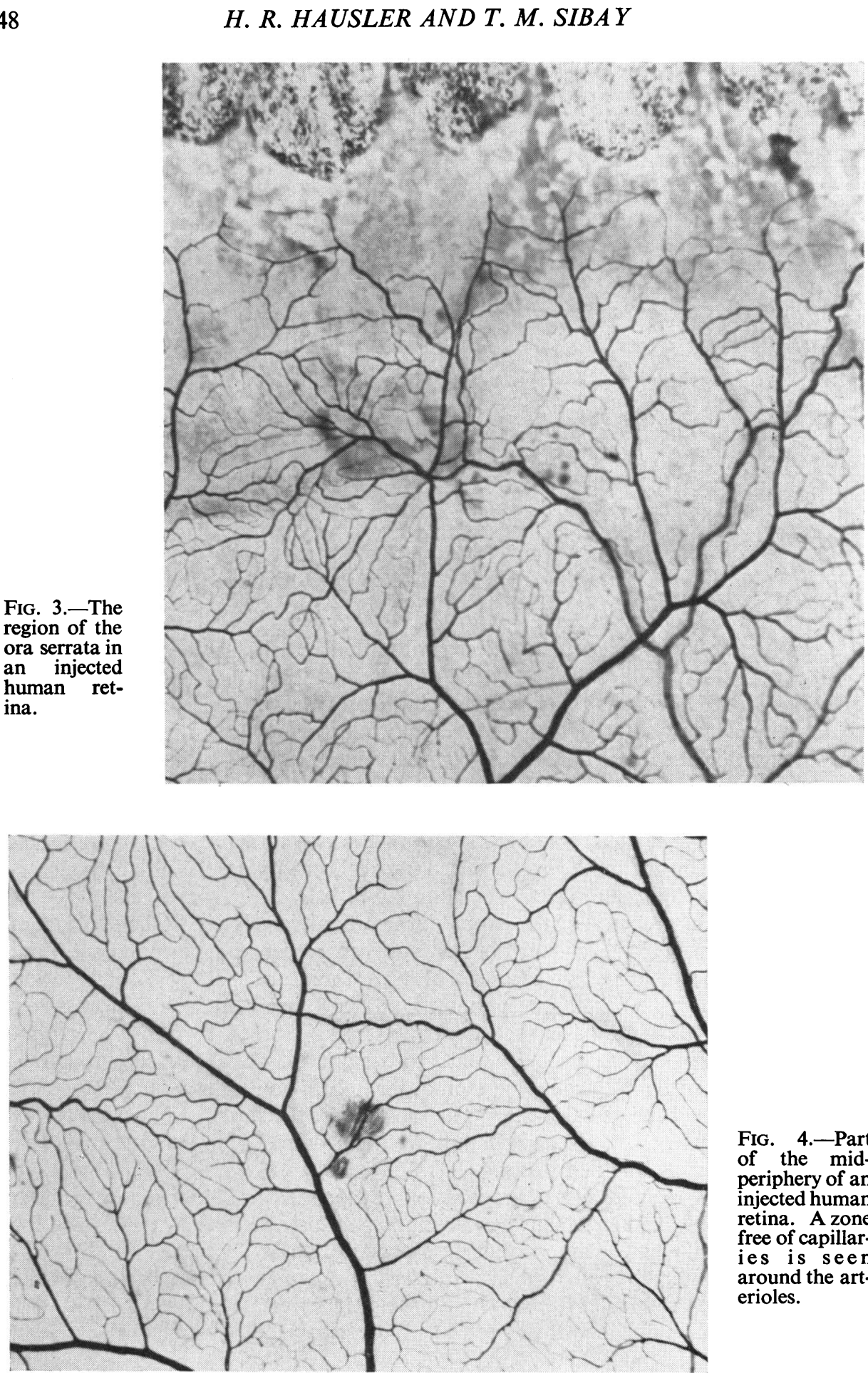

Fig. 4.-Part of the midperiphery of an injected human retina. A zone free of capillaries is seen around the arterioles. 


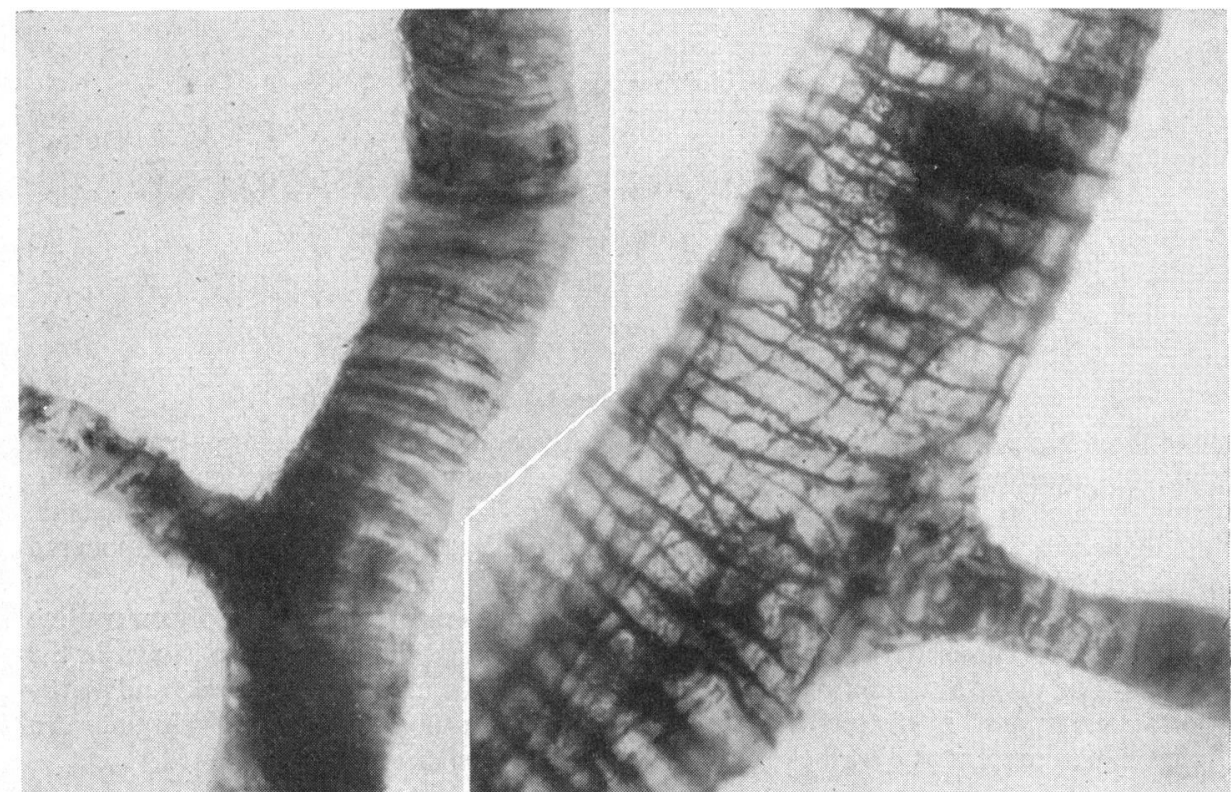

Fig. 5.-Arteriole giving off a capillary. Structural details are evident in the walls of these vessels. A marked radial pattern seen on the wall of the arteriole is less pronounced on the

FIG. 6.-Capillary ending in a venule. These vessels show an axial pattern of staining fibres in addition to the radial pattern which is so prominent on the arterioles. wall of the capillary.

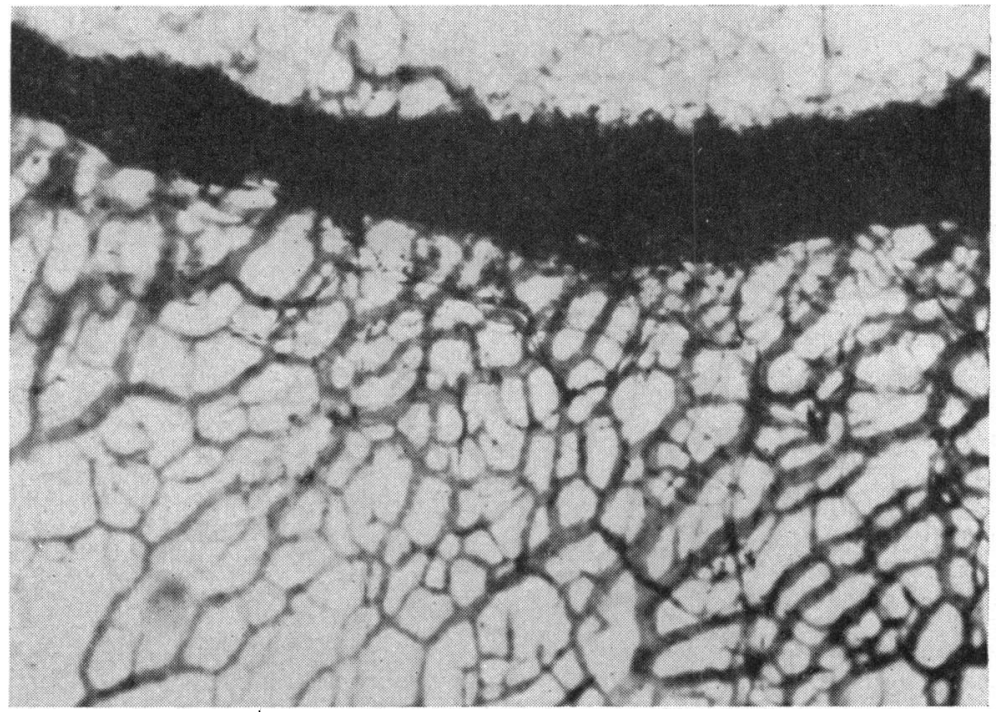

FIG. 7.-Schlemm's canal injected by this method. All the visible vessels were filled by emissaries from the canal. The specimen was cleared after the injection.

\section{REFERENCE}

Hausler, H. R., and Sibay, T. M. (1959). Amer. J. Ophthal., 48, Pt. 2, July, p. 138. 